\title{
The geriatric nutritional risk index mediated the relationship between serum uric acid and hypertension: a mediation analysis
}

\author{
Zhongnan $\mathrm{Cao}^{1 \dagger}$, Sui Dai ${ }^{2 \dagger}$ and Xun $\mathrm{Liu}^{3^{*}}$
}

\begin{abstract}
Background: The elevated serum uric acid (SUA) is associated with an increased risk of hypertension and nutritional status. Malnutrition might modify the association of SUA with hypertension. Therefore, the aims of this study were to examine the mediation effect of malnutrition on the association of SUA with the risk of hypertension in Chinese population.

Methods: The study was based on the China Health and Nutrition Survey in 2009. Participants aged $\geq 60$ years with complete analyzed data were eligible. The Geriatric Nutritional Risk Index (GNRI) was calculated by serum albumin (ALB) and BMI. Participants were identified as hypertension if systolic blood pressure $\geq 140 \mathrm{mmHg}$ and/or diastolic blood pressure $\geq 90 \mathrm{mmHg}$ or receiving antihypertensive drug.

Results: There were 2371 participants included in the final analysis. In total, there was a significant mediation effect of the GNRI on the relationship between SUA level with hypertension ( $P<0.001$; OR: 1.096; and $95 \%$ Cl: 1.0481.146). And the proportion mediated was $17.77 \%$. The results stratified by sex were consistent with those of total population. The significant mediation effects of the GNRI were found in the 60-69 years and 70-79 years groups $(P=0.002$ and $0.032 ; O R: 1.099$ and 1.075 ; and $95 \%$ Cl: $1.036-1.165$ and $1.006-1.148$, respectively) but not in the $80-$ 99 years group $(P=0.303)$. The proportions mediated were $16.22 \%$ and $18.36 \%$, respectively.

Conclusions: The GNRI can mediate and account for approximately $17.77 \%$ of the relationship between SUA level and the risk of hypertension. And this mediation effect was fully observed in both males and females, especially in the 60-79 years population.
\end{abstract}

Keywords: Geriatric Nutritional Risk Index, serum uric acid, hypertension, mediation effect

\section{Background}

Hyperuricemia was prevalent worldwide and has adverse effect on the developments of hypertension, diabetes, and dyslipidemia [1]. Therefore, it has been a major public health issue. There were many studies to report that elevated serum uric acid (SUA) level contributed to the increased incidence of hypertension and cardiovascular

\footnotetext{
* Correspondence: liliudazui@126.com

${ }^{\dagger}$ These authors contributed equally to this article.

${ }^{3}$ Department of Ultrasonics, Tianjin Fifth Central Hospital, No 41 Zhejiang Road, 300450 Tianjin, China

Full list of author information is available at the end of the article
}

mortality [2-6]. Although the association of elevated SUA level with incident hypertension was established, it is suggested that several metabolic factors may mediate the pathways from SUA to hypertension [7]. Previous study declared that hyperinsulinemia is associated with the reabsorption of SUA in the obese subjects [8]. Furthermore, fat cells might modify the association of hyperuricemia with hypertension $[8,9]$.

Previous studies reported that elevated SUA level was associated with nutritional status $[10,11]$. Since consumption of purine-rich meat, seafood, and fat could 
increase SUA level, a low SUA level implied inadequate protein and calorie intake [12]. Therefore, it was likely that protein-energy wasting might cause a low SUA level in the malnourished participants. Furthermore, in a recent study, there was a lower SUA level in the malnourished population than the healthy population [13]. On the other hand, nutritional status was identified as an independent predictor of hypertension [11, 14]. Therefore, there might be interplay between SUA level and nutritional status on hypertension.

In view of the mentioned above, we hypothesized that nutritional status might mediate the relationship between SUA level and the risk of hypertension. However, until now, there was no study to investigate the mediation effect of malnutrition on the relationship between SUA level and the development of hypertension. Since the Geriatric Nutritional Risk Index (GNRI) was commonly used to assess the nutritional status in the elderly, this study was designed to examine the mediation effect of the GNRI on the relationship between SUA level and the risk of hypertension in Chinese elderly population. It is expected that this study will provide a new perspective and evidence to prevent and screen hypertension.

\section{Methods}

\section{Data source}

This study used the data of wave 2009 of the China Health and Nutrition Survey (CHNS). As a national large- scale cohort study, the CHNS focused on the changes of health and nutrition, as well as the effects of the social and economic transformation on health and nutritional statuses in China. It was described in detail in the published literature elsewhere [15].

\section{Study population}

In the CHNS, a multistage and random cluster process was used to sample the study population from nine provinces, which were representative in geography, economic development, and health indicators. All participants were interviewed in household to collect information of lifestyle, health status, marriage and birth history, and detailed physical examinations including weight, height, arm and head circumference, and blood pressure. In the CHNS 2009, the biomarker data of 26 fasting blood measures were released including major cardiovascular biomarkers and important nutrition biomarkers, which has been published for an overview of these biomarkers [16].

The inclusion criteria were as follows: who were aged $\geq 60$ years in the time of survey; and who have complete analyzed data, including height, weight, blood pressure, and uric acid. To explore the mediation effect of malnutrition not attributed to diseases on the association of SUA level with hypertension, participants with severe wasting diseases, such as hepatic disease and severe renal insufficiency, were excluded. Overall, 2371 participants were included in the final analysis. This study was approved by the Institutional Review Board of the National Institute for Nutrition and Food Safety, China Center for Disease Control and Prevention, and University of North Carolina at Chapel Hill. Written informed consent was obtained from all subjects.

\section{Measurements}

Physical index including height and weight were measured by the trained health staff or the members of the CHNS following standardized approach. All index were measured for three times. And the averages were used to analysis. Height and weight were employed to calculate body mass index (BMI).

A valid questionnaire was used to collect the data of health behaviors and medical history. The smoking status was checked via a question as follows: Have you ever smoked cigarettes (including hand-rolled or devicerolled)? If respondents answered "never smoked", he or she was identified as no smoking. If respondents answered "yes", he or she was identified as smoking. And the status of alcohol consumption was interviewed as follows: Did you drink beer or any other alcoholic beverage last year? If respondents answered "no", he or she was identified as no alcohol consumption. If respondents answered "yes", he or she was identified as alcohol consumption. All participants were interviewed for their education levels as follows: What is the highest level of education you have attained? According to the responses, education levels were divided into three levels as follows: primary school or none, middle school, and college or above. Physical activities in this study included martial arts, gymnastics, track and field, soccer and basketball, badminton and volleyball, and others (ping pong, Tai Chi, etc.). Each participant was asked for each activity: Do you participate in this activity? If they answered "no" for all these items, physical activity was identified as "no". If they answered "yes" for at least one of these items, physical activity was identified as "yes". The history of diabetes was identified via a question: Has a doctor ever told you that you suffer from diabetes? If the answer was "no", the history of diabetes was identified as "no". If the answer was "yes", the history of diabetes was identified as "yes". Ethnicity was divided into Han and others according to the participants' identification cards.

All participants were supposed to provide the fasting blood samples in wave 2009. The automated biochemistry analyzer (Hitachi 7600, Randox, UK and Kyowa, Japan) was used to assay SUA and serum albumin (ALB) in Beijing central laboratory. 


\section{Definitions of the GNRI and hypertension}

The GNRI was calculated by ALB and weight as follows: GNRI $=[1.489 \times$ albumin $(\mathrm{g} / \mathrm{L})]+[41.7 \times($ actual weight/ ideal weight)] [17]. Ideal body weight was calculated by the Lorentz formula [17]. Sexspecific Lorentz formula was: ideal weight of males = height $-100-[($ height -150$) / 4]$ and ideal weight of females $=$ height $-100-[($ height -150$) / 2.5]$. If actual weight / ideal weight was more than one, actual weight / ideal weight was set to 1 .

After resting in a sitting position for $5 \mathrm{~min}$ and selecting suitable cuff size, blood pressure was measured for three times using standardized mercury sphygmomanometers. Systolic blood pressure (SBP) corresponds with the first Korotkoff sound, and diastolic blood pressure (DBP) corresponds with the fifth Korotkoff sound. And the averages were used to define hypertension as follows: SBP/ DBP $\geq 140$ / $90 \mathrm{mmHg}$ or taking antihypertensive medications [18].

\section{Statistical analysis}

Means \pm standard deviations were used to describe continuous variables. And frequencies (percentages) were used to describe categorical variables. The between-group comparisons were conducted by $t$-test and chi-square test for continuous variables and categorical variables, respectively. Logistic regression was employed to examine the associations of the GNRI and SUA level with hypertension and obtain odd ratios $(O R s)$ and $95 \%$ confidential intervals $(C I s)$. In the adjusted models, age, sex, current smoking, current drinking, education degree, physical activity, ethnicity, and history of diabetes were adjusted. In the mediate analysis, the GNRI, SUA, and hypertension were taken as the mediate variable, independent variable, and response variable, respectively. The Directed Acyclic Graph of relations among these variables is depicted in Fig. 1.
All mediation analyses were conducted using the SAS macro procedure package "mediation analysis" [19]. And the controlled direct effect (CDE), natural direct effect (NDE), natural indirect effect (NIE), and proportion mediated were provided. Based on the counterfactual framework, the CDE is the effect of exposure on outcome as mediator was controlled. The NDE, namely pure direct effect, is the effect of exposure on outcome with mediator was kept at the level it would naturally have taken. The NIE is the effect of mediator on outcome as exposure was controlled. When outcome is binary, the total effect is the overall effect of exposure on outcome regardless of mediator, and equals to odds ratio of the NDE multiplying by odds ratio of NIE. Similarly, the proportion mediated equals to odds ratio of the NIE divided by odds ratio of total effect. SAS 9.4 (SAS Institute Inc., Cary, NC, USA.) was employed to conduct all analyses. And a two-tailed $P \leq 0.05$ was considered as statistical significance.

\section{Results}

The characteristics of all participants

A total of 2371 subjects (1120 males and 1251 females) were eligible. The mean age was 69.23 years. There were $52.64 \%$ of participants with hypertension. The average of the GNRI was 109.16. The average of SUA level was $5.38 \mathrm{mg} / \mathrm{dl}$. Significant differences between normal and hypertension groups were observed in all characteristics but not sex $(P=0.483)$, current drinking $(P=0.230)$, education degree $(P=0.965)$, and physical activity $(P=$ 0.780) (Table 1).

\section{The associations of the GNRI and SUA level with hypertension}

The associations of the GNRI and SUA level with hypertension are shown in Table 2. In model 1, the GNRI was associated with a higher risk of hypertension $(P<0.001$; OR: 1.060; and $95 \%$ CI: 1.045-1.076). In model 2, there was positive association of SUA level with hypertension

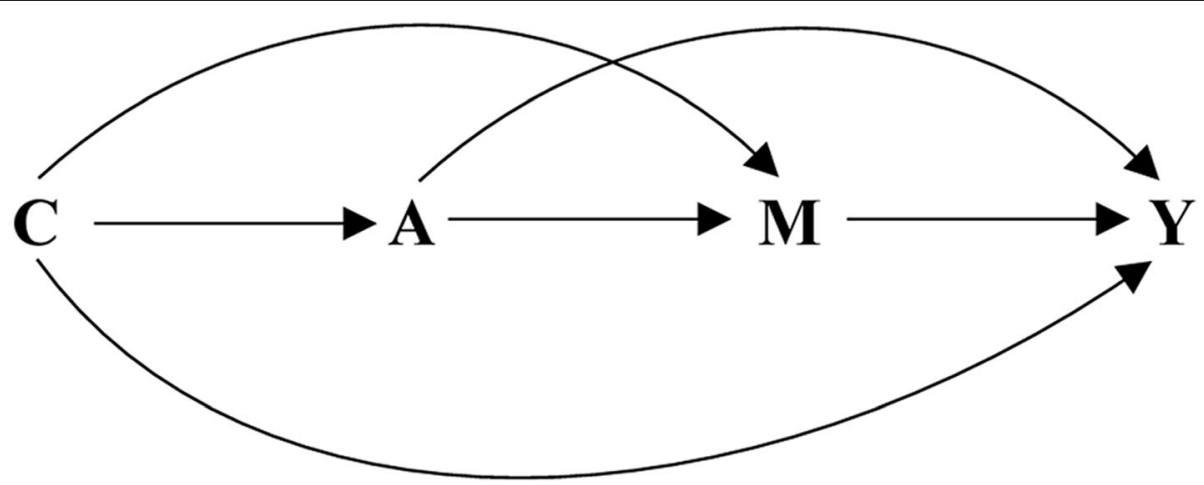

Fig. 1 The Directed Acyclic Graph of exposure, mediation, and outcome. $A=$ exposure; $M=$ mediator; $Y=$ outcome; $C=c o v a r i a t e s$ 
Table 1 The characteristics of all subjects

\begin{tabular}{|c|c|c|c|c|c|}
\hline \multirow[t]{2}{*}{ Characteristics } & \multirow{2}{*}{$\begin{array}{l}\text { All subjects } \\
(2371)\end{array}$} & \multicolumn{2}{|l|}{ Subgroup subjects } & \multirow[t]{2}{*}{$t / x^{2}$} & \multirow[t]{2}{*}{$P$} \\
\hline & & Non-hypertension (1123) & Hypertension (1248) & & \\
\hline Age $\left(\right.$ years) ${ }^{*}$ & $69.23 \pm 6.77$ & $68.30 \pm 6.59$ & $70.06 \pm 6.83$ & -6.360 & $<0.001$ \\
\hline BMI $\left(\mathrm{kg} / \mathrm{m}^{2}\right)^{*}$ & $23.29 \pm 3.68$ & $22.44 \pm 3.38$ & $24.05 \pm 3.77$ & -10.950 & $<0.001$ \\
\hline SUA $(\mathrm{mg} / \mathrm{dl})^{*}$ & $5.38 \pm 1.54$ & $5.14 \pm 1.45$ & $5.59 \pm 1.60$ & -7.290 & $<0.001$ \\
\hline GNRI ${ }^{*}$ & $109.16 \pm 6.33$ & $108.14 \pm 6.31$ & $110.07 \pm 6.20$ & -7.510 & $<0.001$ \\
\hline $\operatorname{Sex}^{\#}$ & & & & 0.493 & 0.483 \\
\hline Male & $1120(47.24)$ & $539(48.00)$ & $581(46.55)$ & & \\
\hline Female & $1251(52.76)$ & $584(52.00)$ & $667(53.45)$ & & \\
\hline Current smoking ${ }^{\#}$ & & & & 4.667 & 0.031 \\
\hline No & 1760(74.26) & $811(72.22)$ & $949(76.10)$ & & \\
\hline Yes & $610(25.74)$ & $312(27.78)$ & $298(23.90)$ & & \\
\hline Current drinking ${ }^{\#}$ & & & & 1.441 & 0.230 \\
\hline No & 1752(73.89) & $817(72.75)$ & 935 (74.92) & & \\
\hline Yes & $619(26.11)$ & $306(27.25)$ & $313(25.08)$ & & \\
\hline Education degree & & & & 0.071 & 0.965 \\
\hline Primary school or none & 1695(71.73) & $801(71.52)$ & 894(71.92) & & \\
\hline Middle school & $596(25.22)$ & $284(25.36)$ & $312(25.10)$ & & \\
\hline College or above & $72(3.05)$ & $35(3.13)$ & $37(2.98)$ & & \\
\hline Ethnicity $^{\#}$ & & & & 12.497 & $<0.001$ \\
\hline Han & 2082(87.81) & $958(85.31)$ & $1124(90.06)$ & & \\
\hline Other & 289(12.19) & 165 (14.69) & $124(9.94)$ & & \\
\hline Physical activity ${ }^{\#}$ & & & & 0.078 & 0.780 \\
\hline No & 2160(91.10) & $1025(91.27)$ & 1135 (90.95) & & \\
\hline Yes & $211(8.90)$ & $98(8.73)$ & $113(9.05)$ & & \\
\hline Diabetes history ${ }^{\#}$ & & & & 23.710 & $<0.001$ \\
\hline No & 1829(77.14) & 916 (81.57) & 913 (73.16) & & \\
\hline Yes & $542(22.86)$ & 207 (18.43) & 335 (26.84) & & \\
\hline
\end{tabular}

※These variables were analyzed using $t$ test

"These variables were analyzed using chi-square test

BMI: body mass index, GNRI: Geriatric Nutritional Risk Index, SUA: serum uric acid

Table 2 The associations of the GNRI and SUA with the risk of hypertension

\begin{tabular}{lllll}
\hline Factors & $\boldsymbol{\beta}$ & $\boldsymbol{P}$ & OR & $\mathbf{9 5 \% \mathrm { Cl }}$ \\
\hline $\begin{array}{l}\text { Model } 1 \\
\text { GNRI }\end{array}$ & 0.059 & $<0.001$ & 1.060 & $1.045-1.076$ \\
Model 2 & & & & \\
SUA & 0.330 & $<0.001$ & 1.391 & $1.243-1.558$ \\
Model 3 & & & & \\
GNRI & 0.054 & $<0.001$ & 1.055 & $1.039-1.071$ \\
SUA & 0.269 & $<0.001$ & 1.309 & $1.167-1.468$ \\
\hline
\end{tabular}

GNRI: Geriatric Nutritional Risk Index, SUA: serum uric acid
$(P<0.001 ;$ OR: 1.391 ; and $95 \% C I: 1.243-1.558)$. When the GNRI and SUA level were analyzed simultaneously, the result was comparable (all $P<0.001 ; O R$ : 1.055 and 1.309; and $95 \%$ CI: $1.039-1.071$ and 1.167-1.468, respectively).

\section{The mediation effect of the GNRI on the relationship between SUA level and hypertension}

Table 3 shows the mediation effect of the GNRI on the relationship between SUA level and hypertension. In total population, there was a significant mediation effect $(P<0.001 ;$ OR: 1.096; and $95 \% C I$ : 1.048-1.146). And the proportion mediated was $17.77 \%$. Meanwhile, both 
Table 3 The mediation effect of the GNRI on the association of SUA with the risk of hypertension

\begin{tabular}{|c|c|c|c|c|}
\hline Path & $O R$ & $P$ & $95 \% \mathrm{Cl}$ & Proportion mediated (\%) \\
\hline \multicolumn{5}{|c|}{ Whole sample $(N=2371)$} \\
\hline CDE & 1.799 & $<0.001$ & $1.604-2.017$ & - \\
\hline NDE & 1.799 & $<0.001$ & $1.430-2.262$ & - \\
\hline NIE & 1.096 & $<0.001$ & $1.048-1.146$ & 17.77 \\
\hline Total effect & 1.971 & $<0.001$ & $1.562-2.488$ & - \\
\hline \multicolumn{5}{|l|}{$\begin{array}{l}\text { Males } \\
(N=1120)\end{array}$} \\
\hline CDE & 1.717 & $<0.001$ & $1.417-2.080$ & - \\
\hline NDE & 1.717 & 0.006 & $1.170-2.519$ & - \\
\hline NIE & 1.094 & 0.016 & $1.017-1.177$ & 18.36 \\
\hline Total effect & 1.878 & 0.001 & $1.273-2.771$ & - \\
\hline \multicolumn{5}{|l|}{$\begin{array}{l}\text { Females } \\
(N=1251)\end{array}$} \\
\hline CDE & 1.802 & $<0.001$ & $1.559-2.083$ & - \\
\hline NDE & 1.802 & $<0.001$ & $1.349-2.407$ & - \\
\hline NIE & 1.093 & 0.001 & $1.035-1.155$ & 17.33 \\
\hline Total effect & 1.970 & $<0.001$ & $1.469-2.642$ & - \\
\hline
\end{tabular}

GNRI: Geriatric Nutritional Risk Index, CDE: controlled direct effect, NDE: natural direct effect, NIE: natural indirect effect, SUA: serum uric acid

in males and females, the results were in line with those of the total population $(P=0.016$ and $0.001 ;$ OR: 1.094 and 1.093; and $95 \% C I: 1.017-1.177$ and 1.035-1.155, respectively). And the proportions mediated were $18.36 \%$ and $17.33 \%$, respectively.

Table 4 The mediation effect of the GNRI on the association of SUA with the risk of hypertension stratified by age

\begin{tabular}{|c|c|c|c|c|}
\hline Path & $O R$ & $P$ & $95 \% \mathrm{Cl}$ & Proportion mediated (\%) \\
\hline \multicolumn{5}{|c|}{$\begin{array}{l}60-69 \text { years } \\
(N=1403)\end{array}$} \\
\hline CDE & 2.038 & $<0.001$ & $1.754-2.368$ & - \\
\hline NDE & 2.038 & $<0.001$ & $1.510-2.752$ & - \\
\hline NIE & 1.099 & 0.002 & $1.036-1.165$ & 16.22 \\
\hline Total effect & 2.239 & $<0.001$ & $1.650-3.038$ & - \\
\hline \multicolumn{5}{|c|}{$\begin{array}{l}70-79 \text { years } \\
(N=777)\end{array}$} \\
\hline CDE & 1.500 & $<0.001$ & $1.227-1.833$ & - \\
\hline NDE & 1.500 & 0.048 & $1.004-2.240$ & - \\
\hline NIE & 1.075 & 0.032 & $1.006-1.148$ & 18.36 \\
\hline Total effect & 1.612 & 0.021 & $1.076-2.414$ & - \\
\hline \multicolumn{5}{|c|}{$\begin{array}{l}80-99 \text { years } \\
(N=191)\end{array}$} \\
\hline CDE & 1.410 & 0.113 & $0.922-2.155$ & - \\
\hline NDE & 1.410 & 0.428 & $0.603-3.293$ & - \\
\hline NIE & 1.094 & 0.303 & $0.922-1.297$ & 24.42 \\
\hline Total effect & 1.542 & 0.324 & $0.652-3.645$ & - \\
\hline
\end{tabular}

GNRI: Geriatric Nutritional Risk Index, CDE: controlled direct effect, NDE: natural direct effect, NIE: natural indirect effect, SUA: serum uric acid
Table 4 shows the mediation effects of the GNRI on the relationship between SUA level and hypertension stratified by age. In the 60-69 years and the $70-79$ years groups, significant mediation effects of the GNRI were found $(P=0.002$ and $0.032 ; O R: 1.099$ and 1.075; and $95 \%$ CI: $1.036-1.165$ and 1.006-1.148, respectively) but not in the $80-99$ years $(P=0.303)$. And the proportions mediated were $16.22 \%$ and $18.36 \%$ in the $60-69$ years and the 70-79 years groups, respectively.

\section{Discussion}

In this study, we found that the GNRI mediated the relationship between SUA level and hypertension. And the mediation effect was fully observed in the 60-79 years population. The proportion mediated by malnutrition was approximately $17.77 \%$.

It has been confirmed that elevated SUA level contributed to the development of hypertension.[20, 21] But the contribution of nutritional status was not taken into account in these studies. A recent study found that waist circumference and BMI, as obesity indicators, may mediate the relationship between SUA level and hypertension.[7] Several studies have examined the associations of abdominal obesity and visceral fat with SUA level.[22, 23] A recent study reported that a higher BMI was significantly associated with an increased risk of new-onset hyperuricemia among hypertensive patients.[24] Therefore, nutritional status might mediate the association of SUA level with hypertension through the path of BMI. Furthermore, a review declared that SUA levels were associated with adverse outcomes in the general 
population, including incident coronary heart disease, heart failure, atrial fibrillation, and mortality [25]. Besides cardiovascular diseases, it was documented that SUA may be a risk factor for cerebrovascular diseases including Alzheimer's disease and Parkinson's dementia, as well as a possible marker of malnutrition [26]. Therefore, malnutrition defined by the GNRI may modify the association of SUA level with hypertension.

On the other hand, SUA is the main final product of the common pathway of purine nucleotides metabolism, [27] and a naturally occurring antioxidant in blood [28]. An overwhelming amount of studies evidenced that the antioxidant property of ALB can inhibit production of free hydroxyl radicals and scavenge peroxy radicals [29-31]. Moreover, it has been proposed that albumin is a major known antioxidant in extracellular fluids [32]. As a result, SUA level can affect serum albumin, which is one of elements to define the GNRI. Furthermore, previous studies found that a decreased serum albumin level could predict the incidence of hypertension [33-35]. Therefore, the GNRI might also mediate the relationship between SUA level and hypertension by the path of serum albumin. Another study reported that the GNRI was associated with all-cause mortality, which can partly attribute to hypertension [36]. Therefore, there was a significant association of malnutrition with hypertension. This implied that SUA level increased the risk of hypertension partly via the GNRI. Taken together, the GNRI served as a mediator of the relationship between SUA level and hypertension by the paths of ALB and BMI.

In this study, the total effect equals to odds ratio of the NDE multiplying by odds ratio of the NIE. In the total population, odds ratio of the total effect was 1.971. Thus, the total effect of the GNRI and SUA level will result in approximately double risk of hypertension. Furthermore, odds ratio of the indirect effect of malnutrition was 1.096, which meant that the risk of hypertension will increase $9.6 \%$ through the path of the GNRI. Generally, both of the ratio of NIE and NDE and the proportion mediated were used to assess the mediation effect. However, since the proportion mediated is suggested to be more stable than the ratio, the proportion mediated was used to assess the mediation effect of the GNRI in this study [37]. The mediation effect of the GNRI accounted for $17.77 \%$ of the total effect. Therefore, the GNRI considerably mediated the association of SUA level with the risk of hypertension. Thus, if nutrition status was kept under the normal condition by controlling SUA level, the risk of hypertension would be reduced by $17.77 \%$. In this study, the potential cut-off point of SUA was $5.47 \mathrm{mg} / \mathrm{dl}$ according to the GNRI. Therefore, it implied that controlling SUA level below $5.47 \mathrm{mg} / \mathrm{dl}$ was better to prevent from hypertension.
Since this study was a cross-sectional study, it was poor evidence to conclude that the GNRI acted as a causal mediator of the relationship between SUA level and hypertension. However, a previous study reported that nutritional status modified the relationship between SUA level and CVD mortality [38]. Given hypertension is a major and independent risk factor of CVD mortality, the mediation effect of the GNRI might be explicable for the association of SUA level with hypertension [39]. The potential mechanisms might be as follows: First, the concentration of SUA was strongly associated with the protein and calorie intake, protein-energy wasting in the malnourished participants will result in a low SUA level [38]. Second, a low SUA level in the malnourished participants might be similar with deficiency of vitamins $C$ and D, both of which are antioxidant and antiinflammatory vitamins [40]. Therefore, a low SUA level would lead to vascular smooth muscle cell proliferation, inflammation, and oxidative stress, as well as rise blood pressure [41]. Third, since the antioxidant property and large antioxidant capacity of SUA, a low SUA level would increase blood pressure due to the decreased antioxidant capacity in the malnourished participants [42]. Taken together, we declared that the GNRI was a mediator of the association of SUA level with hypertension.

In this study, the GNRI defined by BMI and ALB was taken as a mediator but not SUA. Although the direction of the relationship between SUA level and the GNRI failed to be completely clear, there were some indirect evidences. Previous study suggested that SUA level may elevate ALB level and cause albuminuria by the paths of the activated renin-angiotensin system, endothelial dysfunction, and low-grade inflammation, which involved in the development of hypertension [43]. Furthermore, hyperuricemia can induce renal vasoconstriction and cortical ischemia to develop into preglomerular arteriolopathy and tubulointerstitial inflammation, which in turn will cause albuminuria and an increase in blood pressure [44]. On the other hand, a longitudinal study demonstrated that a high SUA level increases BMI, which was used to define the GNRI [45]. Therefore, SUA level will affect nutritional status. Given that the mentioned above, the GNRI was suitable to be a mediator of the association of SUA level with hypertension.

\section{Strengths and limitations}

The present study had several strengths. First, due to the good representativeness of this study, the current results were comprehensive and representative of China. Second, the GNRI was defined using BMI and ALB in the present study. Thus, this indicator could fully reflect nutrition status and was more clinically significant. Therefore, the conclusion of this study was accurate and significant. Third, this was the first study to investigate 
the mediation effect of the GNRI on the relationship between SUA level and hypertension. Thus, this study would provide new viewpoints and evidences to prevent from hypertension.

However, there were still limitations to be noted. First, this study was based on a cross-sectional study, which was poor to examine the causal mediation effect. A longitudinal study should be further conducted to investigate the causal mediation effect of the GNRI on the association of SUA with hypertension in the future. Second, the data of dietary and history of cardiovascular diseases were unavailable in the CHNS. Therefore, the confounding effect might not be fully corrected. Third, the mechanisms of the GNRI mediating the association of SUA level with hypertension failed to be fully explained. The underlying mechanism needs to be further investigated in the laboratory. Fourth, the sample size of the 80-99 years group was not enough, which might contribute to the disappeared mediation effect of the GNRI on the relationship between SUA level and hypertension.

\section{Conclusions}

This study provided epidemiological data to show that there was significant mediation effect of the GNRI on the relationship between SUA level and hypertension. And this mediation effect was fully observed in both males and females, especially in the 60-79 years population. Furthermore, the GNRI can account for approximately $17.77 \%$ of the relationship between SUA level and hypertension.

\begin{abstract}
Abbreviations
SUA: Serum uric acid; GNRI: Geriatric Nutritional Risk Index; CHNS: China Health and Nutrition Survey; BMI: Body mass index; ALB: Albumin; SBP: Systolic blood pressure; DBP: Diastolic blood pressure; ORs: Odd ratios; Cls: Confidential intervals; CDE: Controlled direct effect; NDE: Natural direct effect; NIE: Natural indirect effect
\end{abstract}

\section{Acknowledgements \\ This research uses data from China Health and Nutrition Survey (CHNS). We thank the National Institute for Nutrition and Health, China Center for Disease Control and Prevention, Carolina Population Center, the University of North Carolina at Chapel Hill, the NIH and the NIH Fogarty International Center for the CHNS data collection and analysis files from 1989 to 2015 and future surveys, and the China-Japan Friendship Hospital, Ministry of Health for support for CHNS 2009, Chinese National Human Genome Center at Shanghai since 2009, and Beijing Municipal Center for Disease Prevention and Control since 2011.}

\section{Authors' contributions}

ZC analyzed and interpreted the data. SD was a major contributor in writing the manuscript. XL contributed to the design of this study and revised the manuscript. All authors read and approved the final manuscript.

\section{Funding}

Not applicable.

\section{Availability of data and materials}

The datasets analyzed during the current study are available in the CHNS: http://www.cpc.unc.edu/projects/china.

\section{Declarations}

\section{Ethics approval and consent to participate}

This study was performed in accordance with the Declaration of Helsinki and was approved by the Institutional Review Board of the National Institute for Nutrition and Food Safety, China Center for Disease Control and Prevention, and University of North Carolina at Chapel Hill. Written informed consent was obtained from all subjects.

Consent for publication

Not applicable.

\section{Competing interests}

The authors declare that they have no competing interests.

\section{Author details}

'Department of Cardiology, Tianjin Fifth Central Hospital, 300450 Tianjin, China. ${ }^{2}$ Department of Laboratory, Tianjin Fifth Central Hospital, 300450

Tianjin, China. ${ }^{3}$ Department of Ultrasonics, Tianjin Fifth Central Hospital, No 41 Zhejiang Road, 300450 Tianjin, China.

Received: 22 June 2021 Accepted: 17 September 2021

Published online: 02 October 2021

\section{References}

1. Borghi CR, E. A. Bardin, T. Dawson, J. Dominiczak, A. Kielstein, J. T. Manolis, A. J. et al: Serum uric acid and the risk of cardiovascular and renal disease. Journal of hypertension 2015, 33(9):1729-1741; discussion 1741.

2. Wei F SN, Cai C, Feng S, Tian J, Shi W, et al: Associations between serum uric acid and the incidence of hypertension: a Chinese senior dynamic cohort study. Transl Med 2016, 14(1):110.

3. Kuwabara M HI, Niwa K, Hara S, Roncal-Jimenez CA, Bjornstad P,et al: Uric Acid is a Strong Risk Marker for Developing Hypertension from Prehypertension: A 5-year Japanese Cohort Study. Hypertension 2018, 71(1):78-86

4. Kansui Y MK, Morinaga Y, Inoue M, Kiyohara K, Ohta Y, et al: Impact of serum uric acid on incident hypertension in a worksite population of Japanese men. Hypertens 2018, 36(7):1499-1505.

5. Virdis A MS, Casiglia E, Tikhonoff V, Cicero A F G, Ungar A, et al: Identification of the Uric Acid Thresholds Predicting an Increased Total and Cardiovascular Mortality Over 20 Years. Hypertension 2020, 75(2):302308.

6. Cicero A F SP, D'Addato S, Rosticci M, Borghi C, Brisighella Heart Study group: Association between serum uric acid, hypertension, vascular stiffness and subclinical atherosclerosis: data from the Brisighella Heart Study. Journal of hypertension 2014, 32(1):57-64.

7. Cao Zhi CY, Li Shu,Yang Hongxi,Sun Li,Gao Ying,et al: Mediation of the effect of serum uric acid on the risk of developing hypertension: a population-based cohort study. Journal of translational medicine 2019, 17(1):202

8. Facchini F CY, Hollenbeck CB, Reaven GM: Relationship between resistance to insulin-mediated glucose uptake, urinary uric acid clearance, and plasma uric acid concentration. JAMA 1991, 266(21):3008-3011.

9. Hayashi T BE, Leonetti DL, McNeely MJ, Newell-Morris L, Kahn SE, et al: Visceral adiposity is an independent predictor of incident hypertension in Japanese Americans. Ann Intern Med 2004, 140(12):992-1000.

10. Zheng Rongjiong YT, Chen Qingmei,Chen Changxi,Mao Yushan: Serum Uric Acid Concentrations Can Predict Hypertension: A Longitudinal Population-Based Epidemiological Study. Horm Metab Res 2017, 49(11): 873-879.

11. Cicero A F G FF, Desideri G, Grandi E, Rizzoli E, D'Addato S, et al: Arterial Stiffness, Sugar-Sweetened Beverages and Fruits Intake in a Rural Population Sample: Data from the Brisighella Heart Study. Nutrients 2019, 11(11).

12. H M Loenen HE, M R Löwik, E G Schouten, K F Hulshof, J Odink, et al: Serum uric acid correlates in elderly men and women with special reference to body composition and dietary intake (Dutch Nutrition Surveillance System)」 Clin Epidemiol 1990, 43(12):1297-1303.

13. Saghafi-AsI M V-ME: Comprehensive comparison of malnutrition and its associated factors between nursing home and community dwelling 
elderly: a case-control study from Northwestern Iran. Clin Nutr 2017, 21: 51-58.

14. Cereda E PC, Zagami A, Vanotti A, Piffer S, Faliva M, et al: Nutritional risk, functional status and mortality in newly institutionalised elderly. The British journal of nutrition 2013, 110(10):1903-1909.

15. Popkin BM DS, Zhai F, Zhang B: Cohort Profile: The China Health and Nutrition Survey-monitoring and understanding socio-economic and health change in China, 1989-2011. International journal of epidemiology 2010, 39(6):1435-1440

16. Yan S, Li J, Li S, Zhang B, Du S, Gordon-Larsen P, Adair L, Popkin B: The expanding burden of cardiometabolic risk in China: the China Health and Nutrition Survey. Obes Rev 2012, 13(9):810-821.

17. Bouillanne O MG, Dupont C, Coulombel I, Vincent JP, Nicolis I, et al: Geriatric Nutritional Risk Index: a new index for evaluating at-risk elderly medical patients. Am J Clin Nutr 2005, 82(4):777-783.

18. Chobanian AV BG, Black HR, Cushman WC, Green LA, Izzo JL, et al: The Seventh Report of the Joint National Committee on Prevention, Detection, Evaluation, and Treatment of High Blood Pressure: the JNC 7 report. JAMA 2003, 289(19):2560-2572.

19. Linda Valeri TJV: Mediation analysis allowing for exposure-mediator interactions and causal interpretation: theoretical assumptions and implementation with SAS and SPSS macros. Psychol Methods 2013, 18(2): 137-150.

20. Scheepers L BA, Dagnelie PC, Schram MT, van der Kallen CJH, Henry RMA, et al: Uric acid and blood pressure: exploring the role of uric acid production in The Maastricht study. Hypertens 2017, 35(10):1968-1975.

21. Qu LH JH, Chen JH: Efect of uric acid-lowering therapy on blood pressure: systematic review and meta-analysis. Ann Med 2017, 49(2):142156.

22. Sumbul HE KA: The abdominal aortic intima-media thickness increases in patients with primary hyperparathyroidism. Exp Clin Endocrinol Diabetes 2018, 127(6):387-395.

23. Gu D DY, Zhao Y, Miao S, Qu Q: Positively increased visceral adiposity index in hyperuricemia free of metabolic syndrome. Lipids Health Dis 2018, 17(1):101.

24. Li Q, Li R, Zhang S, Zhang Y, Liu M, Song Y, Liu C, Liu L, Wang X, Wang B et al: Relation of BMI and waist circumference with the risk of new-onset hyperuricemia in hypertensive patients. QJM 2021. doi: https://doi.org/10.1 093/ajmed/hcaa346.

25. Wu AH, Gladden JD, Ahmed M, Ahmed A, Filippatos G: Relation of serum uric acid to cardiovascular disease. Int I Cardiol 2016, 213:4-7.

26. Tana C, Ticinesi A, Prati B, Nouvenne A, Meschi T: Uric Acid and Cognitive Function in Older Individuals. Nutrients 2018, 10(8): 975.

27. Su Z CZ, Xiang Y, Wang B, Huang Y, Yang D, et al: Low serum levels of uric acid and albumin in patients with Guillain-Barre syndrome. Medicine (Baltimore) 2017, 96(15):e6618.

28. So A TB: Uric acid transport and disease.. J Clin Invest 2010, 120(6):17911799.

29. Marx G CM: Site-specific modification of albumin by free radicals. Reaction with copper(II) and ascorbate.. Biochem J 1986, 236(2):397-400.

30. B H: Albumin -an important extracellular antioxidant?Biochem Pharmacol 1988, 37(4):569-571.

31. Wayner DD BG, Ingold KU, Locke S: Quantitative measurement of the total, peroxyl radical-trapping antioxidant capability of human blood plasma by controlled peroxidation. The important contribution made by plasma proteins. FEBS Lett 1985, 187(1):33-37.

32. Roche M RP, Singh NR: The antioxidant properties of serum albumin. FEBS Lett 2008, 582:1783-1787.

33. Oda E: Decreased Serum Albumin Predicts Hypertension in a Japanese Health Screening Population. Intern Med 2014, 53(7):655-660.

34. Oda E: Serum Albumin May Prevent Hypertension by Inhibiting Angiotensin Converting Enzyme.. Intern Med 2014, 53(20):2411.

35. Kinoshita Hiroyuki AT: A possible role of decreased human serum albumin in the endothelial dysfunction caused by albuminuria. $J$ Hypertens 2018, 36(9):1947-1948.

36. Isao Nishi YS, Yoshie Hamada-Harimura,Masayoshi Yamamoto,Tomoko Ishizu,Akinori Sugano,et al: Geriatric nutritional risk index predicts allcause deaths in heart failure with preserved ejection fraction. ESC Heart Failure 2019, 6(2):396-405.

37. Mackinnon D P WG, Dwyer J H: A Simulation Study of Mediated Effect Measures. Multivariate behavioral research 1995, 30(1):41.
38. Tseng W C CYT, Ou S M, Shih C J, Tarng D C, Taiwan Geriatric Kidney Disease Research Group: U-Shaped Association Between Serum Uric Acid Levels With Cardiovascular and All-Cause Mortality in the Elderly: The Role of Malnourishment. Journal of the American Heart Association 2018, 7(4):e007523.

39. Majid Ezzati, Stephen Vander Hoorn, Carlene M M Lawes, Rachel Leach, W Philip T James, Alan D Lopez, et al: Rethinking the "diseases of affluence" paradigm: global patterns of nutritional risks in relation to economic development. PLoS Med 2005, 2(5):e133.

40. Peng $H L H$, Li C, Chao X, Zhang Q, Zhang Y: Association between vitamin $D$ insufficiency and elevated serum uric acid among middle-aged and elderly Chinese Han women. PloS one 2013, 8(4):e61159.

41. Kestenbaum B KR, de Boer I, Hoofnagle A, Sarnak M J, Shlipak M G, et al: Vitamin D, parathyroid hormone, and cardiovascular events among older adults. Journal of the American College of Cardiology 2011, 58(14): 1433-1441.

42. S R Maxwell HT, D Sandler, C Leguen, M A Baxter, G H Thorpe, et al: Antioxidant status in patients with uncomplicated insulin-dependent and non-insulin-dependent diabetes mellitus. Eur J Clin Invest 1997, 27(6): 484-490.

43. Vaneckova I ML, Behuliak M, Nagelova V, Zicha J, Kunes J: Obesity-related hypertension: possible pathophysiological mechanisms. The Journal of endocrinology 2014, 223(3):R63-78.

44. Johnson R J R-IB, Kang D H, Feig D I, Herrera-Acosta J: A unifying pathway for essential hypertension. American journal of hypertension 2005, 18(3): $431-440$.

45. Zheng R CC, Yang T, Chen Q, Lu R, Mao Y: Serum Uric Acid Levels and the Risk of Obesity: a Longitudinal Population-Based Epidemiological Study. Clinical laboratory 2017, 63(10):1581-1587.

\section{Publisher's Note}

Springer Nature remains neutral with regard to jurisdictional claims in published maps and institutional affiliations.

Ready to submit your research? Choose BMC and benefit from:

- fast, convenient online submission

- thorough peer review by experienced researchers in your field

- rapid publication on acceptance

- support for research data, including large and complex data types

- gold Open Access which fosters wider collaboration and increased citations

- maximum visibility for your research: over $100 \mathrm{M}$ website views per year

At $\mathrm{BMC}$, research is always in progress.

Learn more biomedcentral.com/submissions 